\section{Molecular cloning, expression and regional distribution of rat ciliary neurotrophic factor}

\author{
K. A. Stöckli, F. Lottspeich*, M. Sendtner†, \\ P. Masiakowskił, P. Carroll, R. Götz, D. Lindholm \\ \& H. Thoenen
}

Department of Neurochemistry, Max-Planck-Institute for Psychiatry and *Max-Planck-Institute for Biochemistry, Genecenter. D-8033 Planegg-Martinsried, FRG

CILIARY neurotrophic factor (CNTF) was originally characterized as a survival factor for chick ciliary neurons in vitro ${ }^{1}$. More recently, it was shown to promote the survival of a variety of other neuronal cell types ${ }^{2,3}$ and to affect the differentiation of $\mathrm{E} 7$ chick sympathetic neurons by inhibiting their proliferation and by inducing the expression of vasoactive intestinal peptide immunoreactivity (VIP-IR) 4 . In cultures of dissociated sympathetic neurons from newborn rats, CNTF induces cholinergic differentiation as shown by increased levels of choline acetyltransferase $(\mathrm{ChAT})^{5}$.

† To whom correspondence should be addressed.

† Present address: Regeneron Pharmaceuticals, Inc., Tarrytown, New York, USA.
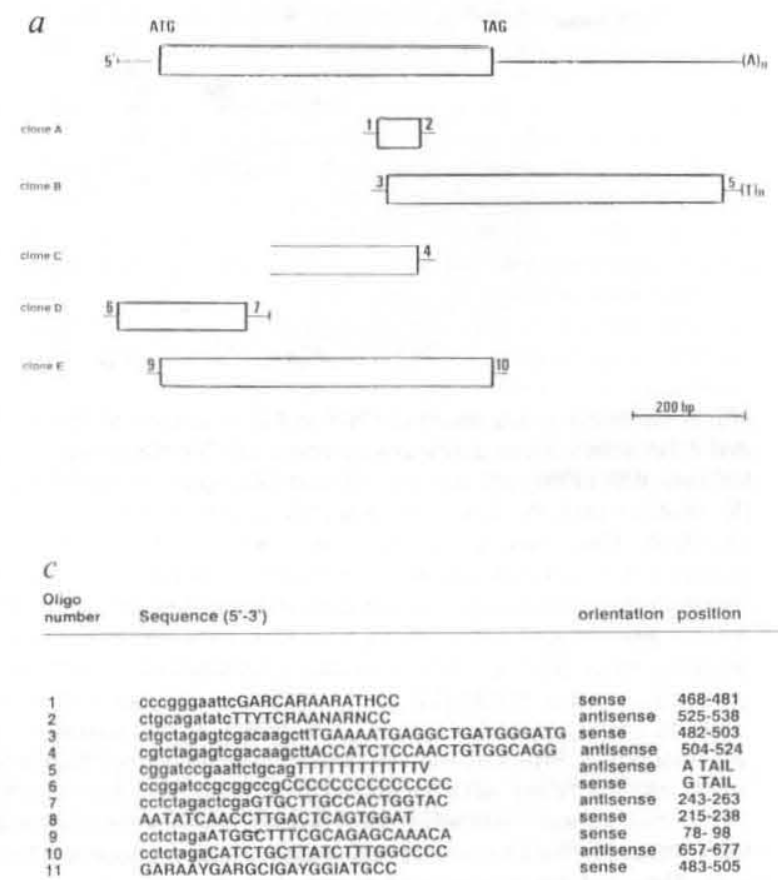

FIG. 1 a, Complementary DNA cloning, and $b$, nucleotide and deduced aminoacid sequence of CNTF. The oligonucleotides used as primers are shown in $c$ and their corresponding positions in the nucleotide sequence in b. Multiple cloning sites are indicated as lower-case letters. In the case of degenerated codons we used IUPAC symbols. Underlined amino acids in $b$ correspond to the peptide sequences obtained from tryptic (T1-T8) and cyanogen bromide (CBI-4) fragments of CNTF. At the nucleotide level, sequences corresponding to the coding region are bold-faced and the polyadenylation signal sequence is underlined.

METHODS. Cultured rat astrocytes were grown for 3 weeks as described ${ }^{20}$ One day after final subculturing, total RNA was extracted from the cells ${ }^{21}$ Complementary DNA was synthesized using oligonucleotide 5 and reverse transcriptase of the Moloney murine leukaemia virus (Bethesda Research Laboratories). The first strand of CDNA served as a template for amplification of specific segments of CNTF using $\mathrm{PCR}^{22}$. Clone A was generated using
This increase is paralleled by a reduction of tyrosine hydroxylase (TH) activity. Moreover, CNTF promotes the differentiation of bipotential 02A progenitor cells to type-2-astrocytes ${ }^{6}$ in vitro. To help establish which, if any, of these functions CNTF exerts in vivo, it is necessary to determine its primary structure, cellular expression, developmental regulation and localization. The complementary DNA-deduced amino-acid sequence and subsequent expression of cDNA clones covering the entire coding region in HeLa-cells indicate that CNTF is a cytosolic protein. This, together with its regional distribution and its developmental expression, show that CNTF is not a target-derived neurotrophic factor. CNTF thus seems to exhibit neurotrophic and differentiation properties only after becoming available either by cellular lesion or by an unknown release mechanism.

CNTF was purified from adult rat sciatic nerve as described previously ${ }^{5}$, using an additional HPLC purification step (for details see legend to Table 1). The amino-acid sequences of the various fragments determined by gas-phase microsequencing represented more than $50 \%$ of the protein. The peptide sequences thus obtained matched perfectly with those deduced from cDNA cloning as shown in Fig. 1.

To obtain RNA for molecular cloning, we used cultures of rat brain cells that previously had been shown to produce substantial quantities of CNTF (ref. 7). After various polymerase chain reaction steps, the nucleotide sequences of clones A, B, $\mathrm{C}$ revealed a short $5^{\prime}$ untranslated region of 77 base pairs (bp) and an open reading frame of $600 \mathrm{bp}$, predicting a protein 200

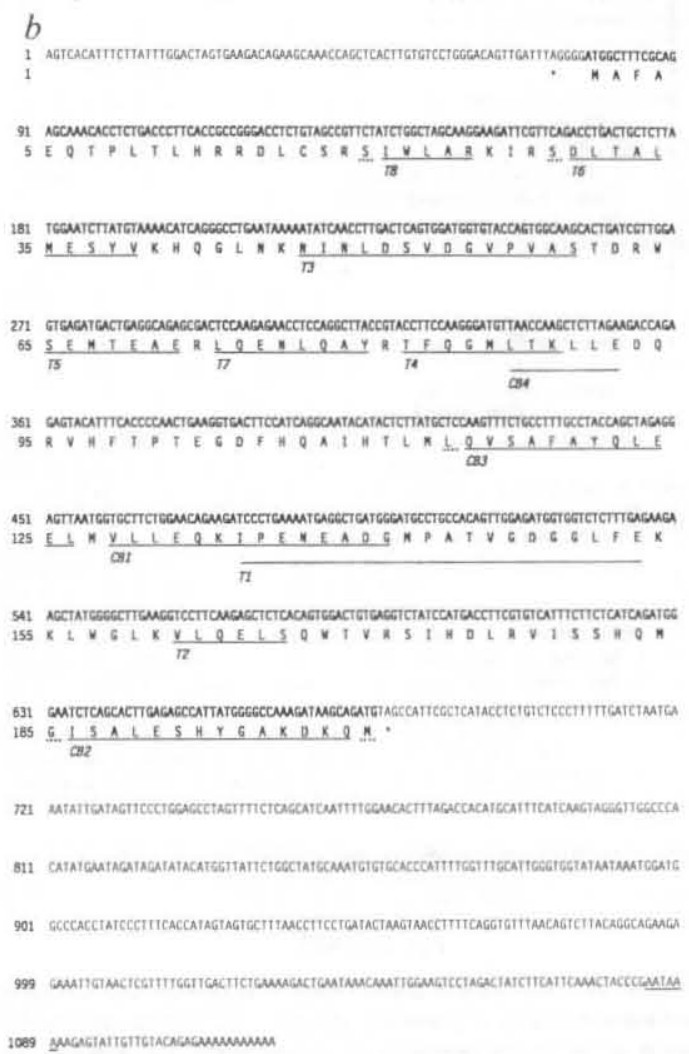

the degenerated primer-oligos 1 and 2; the PCR product was identified with oligonucleotide 11 , subcloned and sequenced by the dideoxynucleotide chaintermination method ${ }^{23}$. The nucleotide sequence thus obtained was used to synthesize primers 3 and 4 for amplification of cDNA ends according to Frohmann et al. ${ }^{24}$ The cDNAs obtained were subcloned into the Bluescript SK + vector (Stratagene) and sequenced (clones B and C). Oligonucleotide 7 was derived from the sequence of a genomic clone (Carroll, unpublished data). This primer, together with oligonucleotide 6 was used to create clone D using essentially the same protocol as for clone C, except that we performed a G-tailing procedure instead of T-tailing to generate 5 'clones ${ }^{25}$ The inserts of several clones were sequenced and shown to be identical. PCR with newly synthesized CDNA from astrocytes and oligonucleotides 9 and 10 revealed a cDNA clone covering the entire coding region. The cDNA thus obtained (Fig. 1a, clone E) was used for expression in eukaryotic cells. 
amino acids long with a $3^{\prime}$ untranslated region of $436 \mathrm{bp}$, which ends in a poly (A) tail (Fig. 1b). The initiation site for translation was localized at position $78-80$ of the nucleotide sequence. A stop codon located $5^{\prime}$ to this initiation site at position 72-74 and $\mathrm{G}$ residues in positions 75 and 81 fulfil the requirements for an adequate translation initiation site, according to $\mathrm{Kozak}^{8}$.

Although the dibasic (Arg-Arg) sequence in positions 13 and 14 of the predicted sequence represents a potential posttranslational cleavage site, the amino-acid composition of purified CNTF (Table 1) does not favour such a cleavage: the amino acids phenylalanine, arginine and alanine present in the $\mathrm{N}$-terminal region are not reduced relative to other amino acids that are absent from this region (for example, isoleucine). Moreover, the predicted relative molecular mass $\left(M_{\mathrm{r}}\right)$ of 22,800 $(22.8 \mathrm{~K})$, corresponding to the 200 -amino-acid sequence is in complete agreement with that estimated from PAGE analysis $(22.5 \mathrm{~K})$ (ref. 6). Thus, the amino-acid sequence of CNTF shows the features of a cytosolic protein; that is, no signal peptide, no consensus sequences for glycosylation and only one cysteine residue at position 17. Comparison of the determined CNTF sequence with those of the PIR and EMBL databases did not reveal significant similarities with any other known protein. In
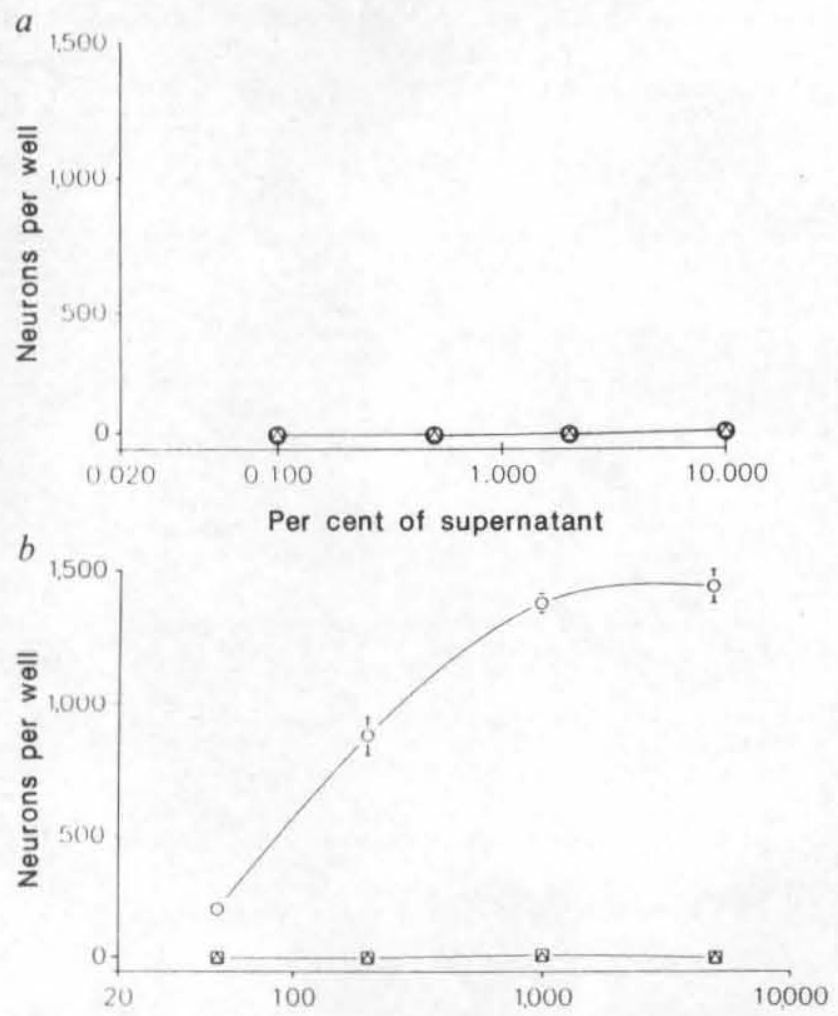

ng extract

FIG. 2 Survival of cultured E8 chick ciliary neurons in the presence of supernatants and extracts of transfected HeLa cells. Embryonic day- 8 ciliary neurons were grown in the presence of supernatants $(a)$ and extracts $(b)$ of HeLa cells transfected with plasmid without insert $(\Delta)$ and plasmid containing CNTF cDNA clone $E$ in sense $(O)$ and antisense orientation ( $\square$ ). METHODS. An expression vector with cytomegalo-promotor (gift of David Russell, Dallas) was used for subcloning a mature CNTF clone (Fig. 1a, clone E) in both orientations. HeLa cells were used for transfections, as no basal expression of survival-promoting activity for embryonic chick ciliary neurons could be detected in these cells. Each culture dish (100-mm diameter) was transfected with $10 \mu \mathrm{g}$ of vector by the DEAE-Dextran method ${ }^{26}$. After $48 \mathrm{~h}$ in culture the supernatants were removed, the cells washed three times with cold PBS buffer and lysed in a $5 \mathrm{mM}$ phosphate buffer containing $30 \mathrm{mM}$ $\mathrm{NaCl}(\mathrm{pH} 7.0)$. After ultracentrifugation $(100,000 \mathrm{~g}, 30 \mathrm{~min})$ of the lysate protein concentrations were determined and different concentrations of the supernatants were added to cultured E8-ciliary neurons. Surviving neurons were counted after $24 \mathrm{~h}$ of culture as described previously ${ }^{5}$. Each point shows the mean of three determinations; bars represent the standard errors.
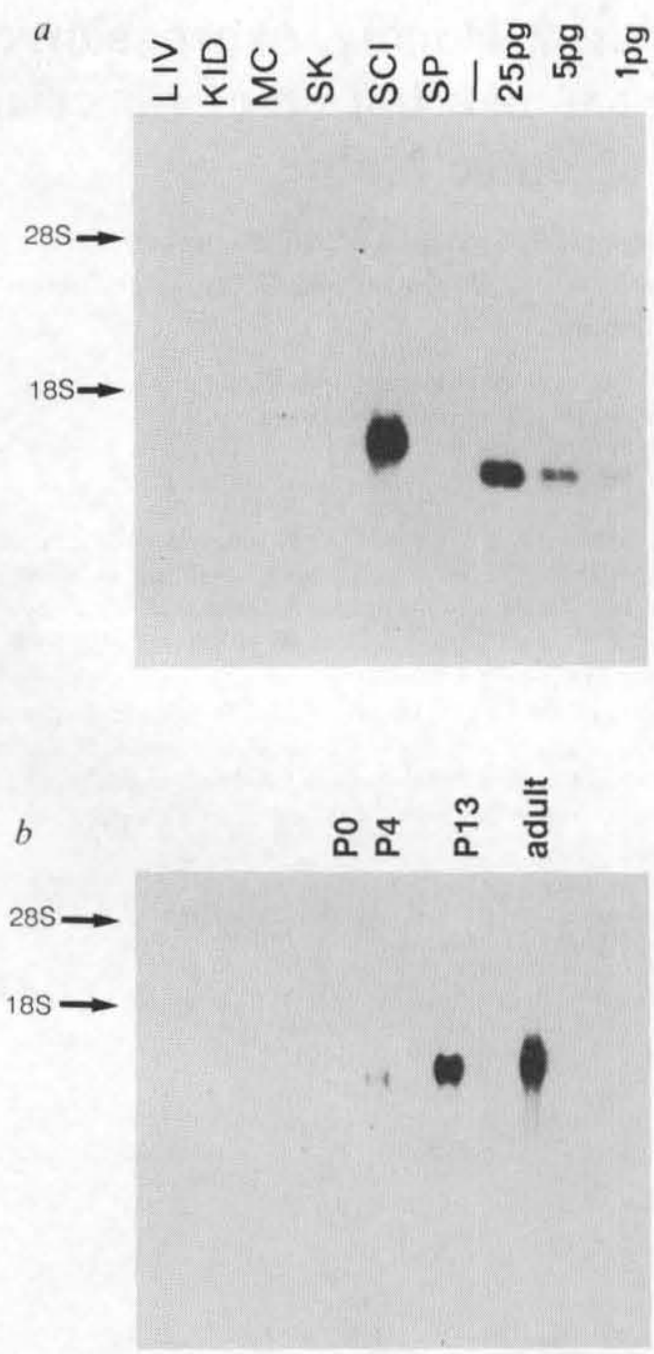

FIG. 3. Northern blot analysis of CNTF mRNA in tissues of the adult rat (a) and in rat sciatic nerve during development $(b)$. The abbreviations used are: LIV, liver; KID, kidney; MC, muscle; SK, skin; SCl, sciatic nerve; SP, spinal cord: $\mathrm{PO}$, newborn rats; P4, 4-day-old rats; P13, 13-day-old rats.

METHODS. RNA was extracted from various rat tissues using the guanidinium thiocyanate extraction method ${ }^{27}$. Total RNA ( $30 \mu \mathrm{g}$, except RNA from muscle which was $50 \mu \mathrm{g}$ ) was glyoxylated and electrophoresed through a $1.2 \%$ agarose $\mathrm{gel}^{28}$. In $b, 25 \mu \mathrm{g}$ total RNA were loaded per lane. Known amounts of a 847-bp CNTF transcript (synthesized in vitro using the riboprobe system, PROMEGA) were also co-electrophoresed in separate lanes to permit quantification of CNTF mRNA in the samples. Following electrophoresis, RNA was vacuum-blotted to nylon filters (Hybond-N, Amersham) and the filters were hybridized at $50^{\circ} \mathrm{C}$ in $50 \%$ formamide 28 using a randomly primed ${ }^{32} \mathrm{P}$-labelled cDNA probe ${ }^{29}$ covering the coding region of CNTF (600 bp). The filters were subsequently washed, exposed for $60 \mathrm{~h}$ to $\mathrm{X}$-ray films and the autoradiograph was photographed.

particular, there was no homology with nerve growth factor (NGF), brain-derived neurotrophic factor (BDNF) ${ }^{9}$ or fibroblast growth factor (FGF) and purpurin, the survival activities of which overlap to some extent with those of $\mathrm{CNTF}^{10,11}$.

The likelihood that CNTF is a cytoplasmic protein is supported by the observation that expression of a cDNA clone in HeLa cells (Fig. 2) resulted in active CNTF being expressed but not being released into the culture medium. We cannot exclude however, that CNTF is secreted by any unconventional release mechanisms, as expression of clone E (Fig. 1a) reveals a Cterminally extended product. CNTF, rather, seems to be a molecule similar to FGF or interleukin-1 (IL-1) both of which exert profound effects on cells but are cytoplasmic proteins. For FGF, no release mechanism has been established ${ }^{12}$. By contrast, IL-1 has been demonstrated to be released from stimulated 
TABLE 1 Amino-acid composition of CNTF

\begin{tabular}{lcc} 
& Purified CNTF & Predicted from cDNA \\
Asx & 17.1 & 16 \\
Thr & 9.9 & 12 \\
Ser & 9.7 & 14 \\
Glx & 30.5 & 30 \\
Pro & n.d. & 5 \\
Gly & 11.7 & 11 \\
Ala & 14.4 & 14 \\
Val & 11.9 & 11 \\
Met & 7.3 & 9 \\
lle & 7.7 & 8 \\
Leu & 24.5 & 26 \\
Tyr & 4.3 & 4 \\
Phe & 6.4 & 6 \\
His & 6.2 & 8 \\
Lys & 10.2 & 10 \\
Arg & 11.1 & 11 \\
Cys & n.d. & 1 \\
Trp & n.d. & 4 \\
\hline
\end{tabular}

Amino-acid composition of CNTF. The amino-acid composition of purified CNTF corresponds to that predicted from the CDNA sequence. Purification and cleavage of CNTF: CNTF was purified as described ${ }^{6}$ but, in addition, after electroelution from preparative polyacrylamide gels, CNTF was applied to a C4-Widepore RP column (Baker, $7.75 \times 100 \mathrm{~mm}$ ) and eluted with $0.1 \%$ trifluoracetic acid and a gradient of acetonitrile. The biologically active protein eluted in one peak at $50-55 \%$ acetonitrile. Two-dimensional gel analysis showed that this protein migrated as a single spot ${ }^{6}$. CNTF obtained by this method was concentrated in a Speedvac-concentrator to a final volume of $50 \mu \mathrm{l}$, diluted to $1 \mathrm{ml}$ with HPLC-grade water and concentrated again to $200 \mu \mathrm{l}$. For cyanogen bromide cleavage, formic acid (final concentration $70 \% \mathrm{v} / \mathrm{v})$ and cyanogen bromide $(10 \% \mathrm{w} / \mathrm{v})$ were added to $30 \mu \mathrm{g}$ of purified CNTF. After $3 \mathrm{~h}$ at room temperature, $500 \mu \mathrm{i} \mathrm{H}_{2} \mathrm{O}$ was added and the material concentrated to $50 \mu \mathrm{l}$ and applied immediately to the same reverse phaseHPLC column. For tryptic cleavage, $30 \mu \mathrm{g} \mathrm{HPLC-purified} \mathrm{CNTF} \mathrm{were} \mathrm{dried,}$ redissolved in $50 \mu \mathrm{l} 0.1 \mathrm{M}$ Tris $\mathrm{HCl}(\mathrm{pH} 8.0)$ containing $10 \mathrm{mM} \mathrm{CaCl}_{2}$ and $3 \mu \mathrm{g}$ tosyl phenylalanine chloromethyl ketone (TPCK)-treated trypsin (Sigma), and incubated overnight at $37^{\circ} \mathrm{C}$. The resulting fragments were loaded on a C4-RP column (Baker) and eluted using the same conditions (flow rate $1 \mathrm{ml} \mathrm{min}{ }^{-1}$, gradient $0-60 \%$ acetonitrile in $60 \mathrm{~min}$, monitoring at $214 \mathrm{~nm}$ ). Peaks were collected manually. The amino-acid sequences of the peptides were determined ${ }^{17}$ by use of automated gas-phase sequencing (Applied Biosystems 470A, 477A). The amino-acid composition of purified CNTF was determined after gas-phase hydrolysis ${ }^{18}$ of $5 \mu \mathrm{g}$ of CNTF on a Beckman 6300 amino-acid analyser. n.d., Not done.

macrophages by an unconventional mechanism after cleavage by a specific enzyme (convertase) ${ }^{13}$. Of particular interest is the recent finding that macromolecules may be exported from the yeast cytosol by carriers that show structural homologies with the multidrug-resistance glycoprotein in mammalian cells ${ }^{14}$. Whether this glycoprotein can also act as a protein carrier in mammalian cells remains to be established.

Northern blot analysis of the distribution of CNTF messenger RNA in tissues of adult rat revealed a single band $\sim 1.2$ kilobases $(\mathrm{kb})$ in size. By far the strongest signal was present in northern blots of the sciatic nerve and a faint band was present in extracts of the spinal cord. But there was no detectable signal in mRNA of muscle and skin; that is, $<2$ pg of CNTF mRNA in $50 \mu \mathrm{g}$ and $30 \mu \mathrm{g}$ of total RNA respectively. The low levels of CNTF mRNA in muscle and skin indicate that the large amount of CNTF present in the sciatic nerve does not represent CNTF transported retrogradely from the periphery-as is the case for NGF-but represents locally synthesized CNTF. Moreover, the developmental time course of CNTF mRNA expression differs from that of $\mathrm{NGF}^{15}$. CNTF mRNA was undetectable in sciatic nerves of newborn rats, only becoming apparent by day 4 (Fig. $3 b$ ). The developmental time course of CNTF mRNA expression indicates that CNTF is not involved in the regulation of neuronal survival in the perinatal period, because target-regulated neuronal cell death ${ }^{16,17}$ is already over by the time the increase in CNTF synthesis begins.

CNTF differs from the known neurotrophic factors NGF and BDNF by the absence of a known constitutive release mechanism, by the time course of its expression during development and by its regional distribution. It may be that CNTF has a physiological role as a differentiation factor, its neurotrophic function possibly only being exerted under pathophysiological conditions rather than during embryonic development.

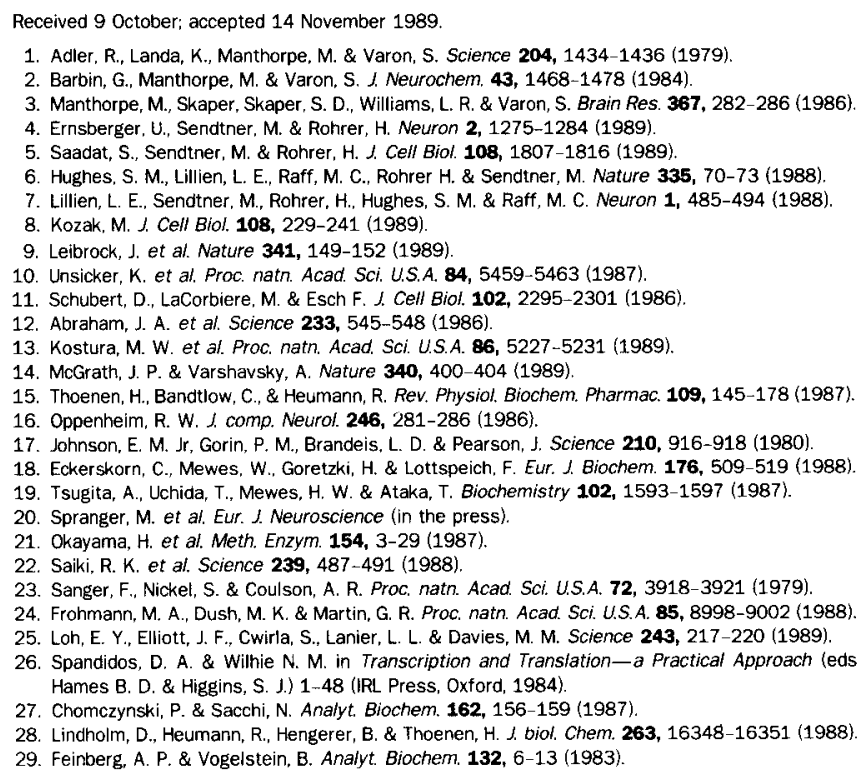

ACKNOWLEDGEMENTS. We thank Yves-Alain Barde for suggestions, David Edgar for critical comments on the manuscript, and Eva Braun for technical assistance. R. G. and P. M. were supported by Regeneron Pharmaceuticals, Inc. Tarrytown, New York. SKA. is recipient of the Stipendienfonds der Basler Chemischen Industrie and the Geigy-Jubiläums-Stiftung. 